\title{
Renormalization and resummation in finite temperature field theories
}

\author{
A. Jakovád \\ Hungarian Academy of Sciences and Budapest University of Technology and \\ Economics Research Group 'Theory of Condensed Matter", H-1521 Budapest, Hungary \\ Zs. Szép \\ Research Group for Statistical Physics of the Hungarian Academy of Sciences, H-1117 Budapest, Hungary
}

\begin{abstract}
Resummation, ie. reorganization of perturbative series, can result in an inconsistent perturbation theory, unless the counterterms are reorganized in an appropriate way. In this paper two methods are presented for resummation of counterterms: one is a direct method where the necessary counterterms are constructed order by order; the other is a general one, based on renormalization group arguments. We demonstrate at one hand that, in mass independent schemes, mass resummation can be performed by gap equations renormalized prior to the substitution of the resummed mass for its argument. On the other hand it is shown that any (momentum-independent) form of mass and coupling constant resummation is compatible with renormalization, and one can explicitly construct the corresponding counterterms.
\end{abstract}

PACS numbers: 11.10.Wx, 11.10.Gh

\section{INTRODUCTION}

Resummation is often required in a perturbation theory to avoid infrared (IR) divergences to appear at finite energy scales. Examples are 1PI diagram resummation (Schwinger-Dyson equation) to cure the on-shell singularity of the propagator, or daisy resummation [1] to resum IR divergences of massless theories at finite temperature. We need leading (or higher order) logarithmic resummation near a second order phase transition point [2], and the HTL resummation [3] to be able to consistently define finite temperature observables in gauge theories.

Resummation means reorganization of the perturbative series in a way that the diagrams that are most important from the point of view of IR behavior, are taken into account first. However, IR importance does not necessarily means UV importance as well, and so it can happen that those (counterterm) diagrams that are needed to make the theory finite, are shifted to a later stage in the resummation process. Then we observe that lower order results are divergent meaning that the resummed perturbation theory is inconsistent. Exceptions are the 1PI and leading log resummations, where, by chance, diagrams of IR and UV importance are the same, but other cases (daisy, superdaisy, HTL etc. resummations) suffer from this difficulty. Therefore, in these cases, the physically well-motivated resummation equations (gap equations) are meaningless in principle, and one usually makes some ad hoc assumption to obtain finite results.

Recently several papers were published that investigate this problem in various physical situations [4]. In the present paper we try to go around the question of how to construct countertem diagrams for a given momentum independent resummation. We try to give two points of view on the problem: one is a direct method where we make explicit resummation for the counterterm diagrams, induced by the resummation of the "normal" diagrams (Section III). The relevant counterterm diagrams can be constructed in each perturbative order, and finally we give an explicit form for the resummed Lagrangian. To be more specific, we suggest, in $\Phi^{4}$ theory, the following reorganization of the mass terms in the Lagrangian in $\overline{\mathrm{MS}}$ scheme to regularize the mass resummation:

$$
-\mathrm{L}_{\text {mass }}=\frac{M_{T}^{2}}{2} \varphi^{2}+\underbrace{\left(-\frac{\Delta M_{T}^{2}}{2} \varphi^{2}+\frac{z \overline{\mathrm{MS}} M_{T}^{2}}{2} \varphi^{2}\right)}_{\text {one loop }}+\underbrace{\left(-\frac{z \overline{\mathrm{MS}} \Delta M_{T}^{2}}{2} \varphi^{2}\right)}_{\text {two loops }},
$$

where $z \overline{\mathrm{MS}}$ is the multiplicative mass renormalization factor in $\overline{\mathrm{MS}}$ scheme, and $M_{T}^{2}=m^{2}+\Delta M_{T}^{2}$. This proposal is a generalization of the method of Banerjee and Mallik [5]. At one hand this expression is equivalent with $\left(m^{2}+\right.$ $\left.z \overline{\mathrm{MS}} m^{2}\right) \varphi^{2} / 2$, so non-perturbatively we have the same physics. On the other hand we will prove that, if we take into

*Electronic address: jakovac@planck.phy.bme.hu

${ }^{\dagger}$ Electronic address: szepzs@achilles.elte.hu 
account the under-braced terms first in one, and two loop levels, respectively, we obtain a mass resummed perturbation theory that consistently removes all the divergencies at any order.

One of the most important corollary of these investigations is the following rule of thumb: we can renormalize the gap equations for mass resummation by first renormalizing the diagrams in an appropriate mass independent physical scheme (eg. $\overline{\mathrm{MS}}$ ), and afterwards substituting the resummed parameters in the finite expressions. As it will be seen later on, although this is not the most generic renormalization method, still it is a possible and consistent way to deal with UV divergences. This strategy was already used in Ref. [6], in the study of phase diagram of the quark-meson model.

The other point of view treats resummed perturbation theory as a different renormalization scheme (Section III). We call this scheme, in which we impose environment $(T, \mu$ or even time) dependent renormalization conditions as resummation $(R S)$ scheme. This is not a physical choice, since changing the environment results in changing of the renormalization scheme. In order to have a meaningful result we have to relate the RS scheme and a bona fide renormalization scheme, eg. $\overline{\mathrm{MS}}$. This means constructing relations between the parameters of the renormalized Lagrangians of the two schemes which formally appear as gap equations.

In several applications we need the inverse procedure: one has a physically motivated gap equation, and one would like to know the corresponding structure of counterterms. In this approach we consider the gap equations as relations between the $\overline{\mathrm{MS}}$ and some RS scheme. Expanding the gap equation in terms of the coupling constant we can read off the finite difference that has to be added to the $\overline{\mathrm{MS}}$ counterterm to obtain the RS scheme counterterm. Thus the RS scheme is defined, and so we can use perturbation theory to calculate any observables we need. If the choice of the gap equations was good enough, we will observe cancellations between the "normal" and counterterm diagrams. The diagrams that survive cancellation are not part of the resummation, and they can be used eventually to improve the resummation process.

The paper ends with conclusions and an outlook (Section $\nabla$ ).

\section{MASS RESUMMATION IN $\overline{\mathrm{MS}}$ SCHEME}

Our basic model is the $\Phi^{4}$ model where the Lagrangian reads in terms of renormalized couplings and fields as

$$
\mathrm{L}=\frac{1}{2}\left(\partial_{\mu} \varphi\right)^{2}-\frac{m^{2}}{2} \varphi^{2}-\frac{\lambda}{24} \varphi^{4}+\frac{Z^{2}}{2}\left(\partial_{\mu} \varphi\right)^{2}-\frac{\delta m^{2}}{2} \varphi^{2}-\frac{\delta \lambda}{24} \varphi^{4} .
$$

As it is well known [1], in this model perturbation theory becomes unreliable at high temperatures, because of the presence of diagrams giving $(T / m)^{N}$ contribution. This (IR) problem can be cured by summing all the dominant tadpole contribution of the theory ("daisy diagrams"). By this procedure one effectively replaces the mass $m^{2}$ by the resummed mass $M_{T}^{2}=m^{2}+\Delta M_{T}^{2}$ (where, in this theory, $\Delta M_{T}^{2}=\frac{\lambda}{24} T^{2}$ to leading order), making the IR problem disappear (apart from the phase transition point [2]).

Since the daisy resummation does nothing else than replacing the mass with a different mass, it is equivalent with the "thermal counterterm" procedure [7]. We add to and subtract from the Lagrangian the same, temperature dependent term; the relevant part of the Lagrangian therefore reads

$$
\frac{m^{2}}{2} \varphi^{2}+\frac{\delta m^{2}}{2} \varphi^{2}=\frac{m^{2}+\Delta M_{T}^{2}}{2} \varphi^{2}-\frac{\Delta M_{T}^{2}}{2} \varphi^{2}+\frac{\delta m^{2}}{2} \varphi^{2},
$$

As usual, the added term is treated as a part of the free Lagrangian while the subtracted term, on the other hand, is taken into account one loop later as a (thermal) counterterm. By doing this we reinterpreted the perturbation theory, ie. we defined a resummation. The value of $\Delta M_{T}^{2}$ can be chosen to be the tadpole value, but its "best value" can be found out from the condition that at one loop order the self-energy is zero

$$
\Pi\left(p=0, M_{T}^{2}\right)-\Delta M_{T}^{2}=0 .
$$

This is a gap equation [8, 9] which is to be solved to find $\Delta M_{T}^{2}$.

It turns out, however, that this naive definition is not consistent [4, 8, 10] because of the mismatch in the counterterm diagrams. To see this we calculate the one loop self energy in the $\Phi^{4}$ theory

$$
\Pi\left(p=0, M_{T}^{2}\right)=m^{2}+\Delta M_{T}^{2}+\frac{\lambda}{2} T_{B}\left(m^{2}+\Delta M_{T}^{2}\right)-\Delta M_{T}^{2}+\delta m^{2},
$$

where $T_{B}$ is the bosonic tadpole function; in dimensional regularization it reads:

$$
T_{B}(M)=\frac{M^{2}}{16 \pi^{2}}\left[-\frac{1}{\varepsilon}+\gamma_{E}-1+\ln \frac{M^{2}}{4 \pi \mu^{2}}\right]+\frac{1}{2 \pi^{2}} \int_{M}^{\infty} d \omega \sqrt{\omega^{2}-M^{2}} n(\omega),
$$


where $n(\omega)$ is the Bose-Einstein distribution.

We should write $T_{B}$ with argument $M=M_{T}$, and that is what creates problem here. We, namely, already renormalized the theory at zero temperature, say, in $\overline{\mathrm{MS}}$ scheme. That fixes the one-loop mass counterterm as

$$
\delta m_{1}^{2}=-m^{2} \frac{\lambda}{32 \pi^{2}}\left[-\frac{1}{\varepsilon}+\gamma_{E}-1-\ln 4 \pi\right] .
$$

So we have a divergency $\sim-M_{T}^{2} / \varepsilon$ and a counterterm $m^{2} / \varepsilon$, that do not cancel each other. There is an unbalanced divergency, and so the RS scheme is inconsistent.

Since at the non-perturbative level (infinite order) the resummed theory is equivalent with the original one (they have the same Lagrangian), we expect that this divergence vanishes by higher loop effects. It has been shown [10] that in the next order (two loop in case of mass) we indeed obtain contributions that cancel this divergence - however, other new unbalanced divergences appear in this order. So finally the perturbation theory will not be consistent at any finite loop level.

\section{A. Resummation of counterterm diagrams}

In order to make the theory consistent, we should find some method to shift up to one loop level those two-loop diagrams that are necessary to cancel the unbalanced divergences at one loop order: ie. we have to reorganize (resum) the counterterm diagrams. The idea is the same as in the case of the thermal mass. We find the correction to the counterterm, add it to and subtract it from the Lagrangian, but classify them to belong to different loop orders. Let us denote the necessary mass term by $\delta m_{T}^{2}$ and call it compensating counterterm. So, instead of (3), we use the following mass terms in the Lagrangian:

$$
\underbrace{\frac{m^{2}+\Delta M_{T}^{2}}{2} \varphi^{2}}_{\text {tree level }}+\underbrace{\left(-\frac{\Delta M_{T}^{2}}{2} \varphi^{2}+\frac{\delta m^{2}+\delta m_{T}^{2}}{2} \varphi^{2}\right)}_{\text {one-loop level }}+\underbrace{\left(-\frac{\delta m_{T}^{2}}{2} \varphi^{2}\right)}_{\text {two-loop level }} .
$$

In perturbation theory the first term is taken into account in the propagator, the second and third are taken into account first at one loop level, while the last term first contributes at two-loop level. $\delta m_{T}^{2}$ has to be determined order by order in a way that it cancels the remaining divergence of the physical observable at a given order. Although it is temperature dependent and also divergent in the present example, its value is irrelevant from the point of view of the consistency of the complete theory, since the Lagrangian is the same as the original one, it does not depend on the value of $\delta m_{T}^{2}$.

Using the proposed decomposition of the Lagrangian corresponding to (8) let us compute the resummed and renormalized self-energy of the $\Phi^{4}$ model.

\section{One loop self-energy}

At one loop level the self-energy reads with this new counterterm structure as

$$
\Pi_{\text {one-loop }}=M_{T}^{2}+\frac{\lambda}{2} T_{B}\left(M_{T}^{2}\right)-\Delta M_{T}^{2}+\delta m_{1}^{2}+\delta m_{T 1}^{2} .
$$

$\delta m_{1}^{2}$ is defined in (7); it cancels a part of the divergence hidden in $T_{B}$, the rest has to be canceled by $\delta m_{T 1}^{2}$. We can choose it in analogy with the $\overline{\mathrm{MS}}$ form (7)

$$
\delta m_{T 1}^{2}=-\Delta M_{T}^{2} \frac{\lambda}{32 \pi^{2}}\left[-\frac{1}{\varepsilon}+\gamma_{E}-1-\ln 4 \pi\right] .
$$

In principle we could add an arbitrary finite term to this form. This would modify the value of $\Delta M_{T}^{2}$, and finally would lead to a different resummation process. In the language of Section III it would correspond to a different renormalization scheme in the zero and finite temperature parts. For further details cf. Section

With this choice, using the form of $T_{B}$ from (6) we find

$$
\Pi_{\text {one-loop }}=m^{2}+\frac{\lambda}{2} T_{B}^{\overline{\mathrm{MS}}}\left(M_{T}^{2}\right)
$$


where

$$
T_{B}^{\overline{\mathrm{MS}}}\left(M^{2}\right)=\frac{M^{2}}{16 \pi^{2}} \ln \frac{M^{2}}{\mu^{2}}+\frac{1}{2 \pi^{2}} \int_{M}^{\infty} d \omega \sqrt{\omega^{2}-M^{2}} n(\omega)
$$

is the $\overline{\mathrm{MS}}$ renormalized tadpole diagram.

We could obtain this result by first renormalizing the divergent diagrams in $\overline{\mathrm{MS}}$ scheme, and afterwards by substituting the resummed mass into the finite expression.

\section{Two loop order}

Now let us turn to the determination of the self-energy at two loop level. The contribution from two-loop level diagrams is represented symbolically in the following way:

$$
\Delta \Pi_{\text {two-loop }}=\frac{\lambda^{2}}{6} \bigcirc+\frac{\lambda^{2}}{4} \bigcirc+\frac{\delta \lambda_{1}}{2} \bigcirc+\frac{\lambda}{2} \bigcirc-\delta m_{T 1}^{2}+\delta m_{2}^{2}+\delta m_{T 2}^{2}-\delta Z_{2} p^{2} .
$$

The propagators have the squared mass $M_{T}^{2}$, in this resummed theory. The value of the diagrams in dimensional regularization, writing out explicitly only their divergent part, are:

$$
\begin{aligned}
& \square=\frac{M_{T}^{2}}{16 \pi^{2}}\left(-\frac{1}{\varepsilon}+\gamma_{E}-1+\ln \frac{M_{T}^{2}}{4 \pi \mu^{2}}\right)+T_{B}^{T \neq 0}, \\
& =\left(-\Delta M_{T}^{2}+\delta m_{1}^{2}+\delta m_{T 1}^{2}\right)\left[\frac{1}{16 \pi^{2}}\left(-\frac{1}{\varepsilon}+\gamma_{E}+\ln \frac{M_{T}^{2}}{4 \pi \mu^{2}}\right)+I_{\text {fish }}^{T \neq 0}\right], \\
& =\frac{3 M_{T}^{2}}{(4 \pi)^{4}}\left[\frac{1}{2 \varepsilon^{2}}-\frac{1}{\varepsilon}\left(\gamma_{E}-\frac{3}{2}+\ln \frac{M_{T}^{2}}{4 \pi \mu^{2}}\right)\right]-\frac{p^{2}}{4(4 \pi)^{4}} \frac{1}{\varepsilon}-\frac{3}{16 \pi^{2}} T_{B}^{T \neq 0} \frac{1}{\varepsilon}+I_{\text {setsun }}^{\text {finite }} \\
& =\frac{M_{T}^{2}}{(4 \pi)^{4}}\left[\frac{1}{\varepsilon^{2}}-\frac{1}{\varepsilon}\left(2 \gamma_{E}-1+2 \ln \frac{M_{T}^{2}}{4 \pi \mu^{2}}\right)\right]-\frac{1}{16 \pi^{2}} \frac{1}{\varepsilon}\left(T_{B}^{T \neq 0}+M_{T}^{2} I_{\text {fish }}^{T \neq 0}\right)+I_{\text {dsc }}^{\text {finite }} .
\end{aligned}
$$

Since we do resummation in the $\overline{\mathrm{MS}}$ scheme, the value of $\delta \lambda_{1}, \delta m_{2}^{2}$ and $\delta Z_{2}$ counterterms are fixed, we cannot modify them. $\delta m_{2}^{2}=z_{m 2} m^{2}$, where

$$
z_{m 2}=\frac{\lambda^{2}}{2(4 \pi)^{4}}\left(\frac{1}{\varepsilon^{2}}-(1-\ln 4 \pi) \frac{1}{\varepsilon}\right)+\text { finite. }
$$

The other counterterms read as

$$
\delta \lambda_{1}=-\frac{3 \lambda^{2}}{32 \pi^{2}}\left(-\frac{1}{\varepsilon}+\gamma_{E}-\ln 4 \pi\right), \quad \delta Z_{2}=\frac{1}{4(4 \pi)^{4}} \frac{1}{\varepsilon} .
$$

Using these values the divergent part is still not canceled, what remains is:

$$
\Delta \Pi_{\text {two-loop }}^{d i v}=-\frac{\lambda^{2} \Delta M_{T}^{2}}{2(4 \pi)^{4}}\left(\frac{1}{\varepsilon^{2}}-(1-\ln 4 \pi) \frac{1}{\varepsilon}\right)+\delta m_{T 2}^{2} .
$$

To cancel this divergency we can choose $\delta m_{T 2}^{2}=z_{m 2} \Delta M_{T}^{2}$, although, of course, an arbitrary finite part could be added to this expression.

With this choice, however, we get further support to the practical observation of the one loop calculation: we first do renormalization in $\overline{\mathrm{MS}}$ scheme, and substitute the resummation mass in the finite expressions. It should be clear, too, that we could add finite terms for $\delta m_{T 1}^{2}$ and $\delta m_{T 2}^{2}$, and then this statement would not be true any more. Thus this is just a - comfortable - possibility. 


\section{B. Higher loop orders}

One can conjecture that this feature persists at higher loop orders, since in $\overline{\mathrm{MS}}$ scheme the overall divergency of a diagram always can be written as $-z_{m} M^{2}$ with some mass-independent $z_{m}$ factor. Then, by choosing $\delta m^{2}=z_{m} m^{2}$ and $\delta m_{T}^{2}=z_{m} \Delta M_{T}^{2}$, the overall divergency disappears. This should also be true for any other mass-independent schemes. We can, therefore, put forward a proposition:

Proposition: If in a renormalization scheme the zero temperature counterterm is $\delta m^{2}=z_{m} m^{2}$ and $z_{m}$ is massindependent, then at finite temperature the theory with thermal counterterm $\Delta M_{T}^{2}$ and compensating counterterm $\delta m_{T}^{2}=z_{m} \Delta M_{T}^{2}$ yields finite result at each order of the perturbation theory.

Proof: Let us consider a 2-point diagram $\mathrm{D}\left(\mathrm{m}^{2}\right)$ in the original (not resummed) theory that has an overall divergence, and all the subdivergences were consistently subtracted. Then, if the theory is renormalizable, the divergence of the diagram should be proportional to $m^{2}$; the proportionality constant can be obtained as $\left.\partial_{m^{2}} \mathrm{D}_{(}\left(m^{2}\right)\right|_{\text {div }}$. Since the divergence is canceled by the counterterm $\delta m^{2}=z_{m} m^{2}$, the divergent part should be equal to an appropriate part from the mass renormalization factor $z_{m}$, which is mass independent

$$
\left.\partial_{m^{2}} \mathrm{D}\right|_{\text {div }}=-z_{\mathrm{D}}
$$

In the resummed theory we have two effects. One is the substitution of the mass term by the resummed mass: $m^{2} \rightarrow m^{2}+\Delta M_{T}^{2}$. The divergent parts of the self energy diagrams, in our mass-independent scheme, are proportional to this new mass term. But we have also prepared the corresponding counterterm, since the third term of (8) reads $\delta m^{2}+\delta m_{T}^{2}=z_{m}\left(m^{2}+\Delta M_{T}^{2}\right)$. So this part of the divergences disappear in the same way as in the unresummed case.

The other effect is the $-\Delta M_{T}^{2}$ insertion coming from the second (thermal) counterterm of (8). The sum of the single mass insertion diagrams is just $-\Delta M_{T}^{2} \partial_{m^{2}} \mathrm{D}\left(m^{2}\right)$, because at each propagator $G^{2}\left(p, m^{2}\right)=\partial_{m^{2}} G\left(p, m^{2}\right)$ (it is evident for Feynman propagators, at finite temperature it can be seen using imaginary time propagators). Its divergent part can be written, using (18), as $\Delta M_{T}^{2} z \mathrm{D}$. This is exactly canceled by the corresponding part of the compensating counterterm at this order: $-\delta m_{T}^{2}=-\Delta M_{T}^{2} z_{m}$.

So at each diagram the overall divergences are canceled by the proposed counterterms. Using BPHZ argumentation, this is enough to make the complete theory finite.

\section{RENORMALIZATION GROUP ANALYSIS}

Now we have a consistent method for how to reorganize the perturbation theory according to a given (mass) resummation in such a way as to have finite results in all orders in $\overline{\mathrm{MS}}$ scheme. In order to generalize the result so as to be able to treat other schemes (not just $\overline{\mathrm{MS}}$ ), or environment dependence of other couplings (cf. vertex resummation), we change our viewpoint, and we rephrase the complete resummation procedure using the renormalization group (RG) argumentation: this will be the topic of the present section.

First we demonstrate at one loop level that we can obtain the results of the previous section using a special renormalization scheme. We define the mass counterterm of the new scheme as

$$
\delta m^{2}=\delta m \frac{2}{\mathrm{MS}}-\Delta M^{2}
$$

where $\delta m \frac{2}{\mathrm{MS}}=z \overline{\mathrm{MS}} m^{2}$ denotes the $\overline{\mathrm{MS}}$ counterterm and $\Delta M^{2}$ is an arbitrary, finite expression. The other counterterms are the same as in $\overline{\mathrm{MS}}$ scheme. Then the one loop self energy reads

$$
\Pi=m^{2}+\frac{\lambda}{2} T_{B}\left(m^{2}\right)+\delta m_{\overline{\mathrm{MS}}}^{2}-\Delta M^{2}
$$

This expression differs in a finite term from the corresponding $\overline{\mathrm{MS}}$ expression. At one hand it assures finiteness, on the other hand this means that the new mass parameter must be different from the $\overline{\mathrm{MS}}$ mass parameter in order to describe the same physics. In fact, applying the ideas of the renormalization theory [11] to the present case, there must be a choice $\mathrm{m}^{2}\left(\mathrm{~m}_{\overline{\mathrm{MS}}}^{2}\right)$ for which we have the same value for all observables in the two schemes at a given order. This function can be read off from the requirement that the bare parameters are the same in the two schemes [1]]:

$$
m^{2}+\delta m^{2}=m_{\frac{2}{\mathrm{MS}}}^{2} \delta m_{\frac{2}{\mathrm{MS}}}^{2}, \quad \Rightarrow \quad m^{2}=m_{\frac{2}{\mathrm{MS}}}^{2}+\Delta M^{2} .
$$


Then the counterterm of the new scheme (19) can be written as

$$
\delta m^{2}=-\Delta M^{2}+z \overline{\mathrm{MS}} m_{\overline{\mathrm{MS}}}^{2}+z \overline{\mathrm{MS}} \Delta M^{2} .
$$

This is the result of the Proposition of subsection $\amalg \mathrm{B}$ applied to the one loop case.

For the definition of the new scheme we need to specify a value for $\Delta M^{2}$. We may, for example, choose the mass shell scheme, where the full self-energy reads $\Pi(p=0)=m^{2}$. From (20) and (21) we find for the value of $\Delta M^{2}$

$$
\frac{\lambda}{2} T_{B}^{\overline{\mathrm{MS}}}\left(m_{\overline{\mathrm{MS}}}^{2}+\Delta M^{2}\right)-\Delta M^{2}=0,
$$

where $T_{B}^{\overline{\mathrm{MS}}}$ now means the tadpole diagram, renormalized in the $\overline{\mathrm{MS}}$ scheme. This equation is the renormalized gap equation for the mass.

This analysis suggests that the mass resummation is equivalent with the choice of a new renormalization scheme. Using the relation of the masses of the two schemes, we can compute finite observables that can be interpreted as the result of the resummed $\overline{\mathrm{MS}}$ scheme. The mass relation, together with the definition of the new scheme yields finite gap equation for the new (resummed) mass, which is the usual gap equation in case of the mass shell scheme.

\section{A. Generalization}

Motivated by the previous subsection, we can start to build up the resummation strategy from the point of view of renormalization schemes. Resummation itself means that some higher order diagrams are taken into account at lower levels with the consequence that these diagrams are missing from the higher order calculation. So, at least for the mass and coupling constant resummation, resummation is equivalent with a scheme that cancels these diagrams with a proper choice of the finite part of the counterterms. Since we play only with the finite part of the counterterms, we will still have a consistent scheme. So this new scheme - the resummation $(R S)$ scheme - differs from a general scheme (eg. $\overline{\mathrm{MS}}$ ) in that a certain set of diagrams is missing from the calculation.

The RS scheme is a bona fide scheme while we keep the counterterms fixed: for example we can equally well use the MS and the $\overline{\mathrm{MS}}$ schemes. However, at finite temperature, the finite parts of the counterterms will depend on temperature, and so the resummation scheme itself varies with temperature. In a more general case RS scheme can depend on the complete environment [12]: beyond the temperature on chemical potential, background condensates, time, etc. Since results in different schemes have different physical interpretation, RS scheme cannot directly describe the effects of the environment variation. Example is the mass shell scheme where $\Pi(p=0)=m^{2}$, and we have no explicit temperature dependence. To be able to draw physical consequences, we have to project the results to a common reference scheme.

Technically what we should do is to relate the RS scheme to a physical renormalization scheme - for concreteness we will use the $\overline{\mathrm{MS}}$ scheme. Since both of them are mathematically correct schemes, in case of renormalizable theories, this can be accomplished by changing the values of renormalized parameters of the Lagrangian [11] (including wave function renormalization). In $\Phi^{4}$ theory there must exist environment-dependent parameters

$$
m=m\left(m_{\overline{\mathrm{MS}}}, \lambda_{\overline{\mathrm{MS}}}, T\right), \quad \lambda=\lambda\left(m_{\overline{\mathrm{MS}}}, \lambda_{\overline{\mathrm{MS}}}, T\right), \quad \zeta=\zeta\left(m_{\overline{\mathrm{MS}}}, \lambda_{\overline{\mathrm{MS}}}, T\right)
$$

such as for any $n$-point function with momenta $\left\{p_{i}\right\}$ we have

$$
G_{\overline{\mathrm{MS}}}^{(n)}\left(p_{i} ; m \frac{\overline{\mathrm{MS}}}{,} \lambda \overline{\mathrm{MS}}\right)=\zeta^{n} G_{\text {resum }}^{(n)}\left(p_{i} ; m, \lambda\right) .
$$

In perturbation theory the equality holds only up to the computed order; in fact the difference is the effect of resummation. The value of the "running" parameters can be computed using the fact that the bare quantities are independent of the scheme [11]. In this way we can use the RS scheme for perturbation theory, enjoying its good IR convergence, and still have results in a physical renormalization scheme (eg. $\overline{\mathrm{MS}}$ ), where also the parameters of the theory can be obtained from the usual renormalization conditions.

Sometimes we can explicitly define the new scheme (eg. the mass shell scheme for mass resummation), but more often we just have a guess for the resummed parameters, and we hope that it performs some kind of resummation. That is we start with explicit functions of the form of (24) for the resummed mass and coupling constant (and eventually, wave function renormalization). According to the line of thought above these relations can be interpreted as generators of a RS scheme: we expand them in power series in $\lambda_{\overline{\mathrm{MS}}}$

$$
m^{2}=m_{\frac{\mathrm{MS}}{2}}+\sum_{n=1}^{\infty} \lambda \frac{n}{\mathrm{MS}} \Delta M_{n}^{2}, \quad \lambda=\lambda_{\overline{\mathrm{MS}}}+\sum_{n=1}^{\infty} \lambda_{\mathrm{MS}}^{n} \Delta \lambda_{n}, \quad \zeta=1+\sum_{n=1}^{\infty} \lambda_{\mathrm{MS}}^{n} \Delta Z_{n},
$$


and we interpret $\Delta M_{n}^{2}$ as the $n$th order finite mass counterterm that has to be added to the $\overline{\mathrm{MS}}$ counterterm; similarly, $\Delta \lambda_{n}$ is the finite coupling constant counterterm, $\Delta Z_{n}$ is the finite wave function renormalization counterterm. Having defined the counterterms, the RS scheme is appropriate to do perturbation theory, and the results can be related to the $\overline{\mathrm{MS}}$ scheme by the same relations (24). If our guess was good enough we will observe cancellation between "normal" and counterterm diagrams at higher orders. The main message, however, is that, independently of whether a choice represents a physically meaningful resummation or not, any choice leads to a finite, consistent perturbation theory.

So as a possible strategy for resummation, we can do the following: we perform regularized perturbation theory in a generic scheme, keeping counterterms free, at a certain loop order. The divergent part of the counterterms are then fixed, as usual, to cancel the UV divergences of the diagrams, while the finite parts are fixed to cancel the IR dangerous parts. So we defined a scheme, which is, however, only a RS scheme, since the counterterms can depend on the environment. We determine its relation to the $\overline{\mathrm{MS}}$ scheme of the form (24) by requiring that the bare parameters should be the same in the two schemes. This relation, by construction, corresponds to a resummation up to this perturbative order, and we should have some plausible guess to choose the higher order terms, hoping that it still performs some kind of resummation. Different choices, of course, correspond to different resummations. This procedure can be repeated at each perturbative order, and so the resummation process can be improved.

\section{COUPLING CONSTANT AND MASS RESUMMATION}

Using the ideas of the previous subsection, in this section we perform both mass and coupling constant resummation. This latter is important to describe the "softening" of the theory close to a phase transition point [2], and also to consistently describe vertex resummation 13].

At one loop level we can start from the effective potential

$$
V=\frac{1}{2} m^{2} \Phi^{2}+\frac{\lambda}{24} \Phi^{4}+\Delta V\left(M^{2}\right)+\frac{1}{2} \delta m^{2} \Phi^{2}+\frac{\delta \lambda}{24} \Phi^{4},
$$

where $M^{2}=m^{2}+\frac{\lambda}{2} \Phi^{2}$. The effective, background dependent mass and coupling constant then reads

$$
\begin{aligned}
& \frac{d^{2} V}{d \Phi^{2}}=M^{2}+\delta m^{2}+\frac{\delta \lambda}{2} \Phi^{2}+\lambda \Delta V^{\prime}\left(M^{2}\right)+\lambda^{2} \Phi^{2} \Delta V^{\prime \prime}\left(M^{2}\right) \\
& \frac{d^{4} V}{d \Phi^{4}}=\lambda+\delta \lambda+3 \lambda^{2} \Delta V^{\prime \prime}\left(M^{2}\right)+\mathrm{Q}\left(\lambda^{3} \Phi^{2}\right)
\end{aligned}
$$

A possible way of doing coupling constant resummation that we make vanish all of the terms below $\mathrm{Q}\left(\lambda^{3} \Phi^{2}\right)$. That means:

$$
\delta \lambda=-3 \lambda^{2} \Delta V^{\prime \prime}\left(M^{2}\right)
$$

For the mass resummation we demand that $V^{\prime \prime}=M^{2}$; with the $\delta \lambda$ value defined above we obtain

$$
\delta m^{2}=-\lambda \Delta V^{\prime}\left(M^{2}\right)+\frac{1}{2} \lambda^{2} \Phi^{2} \Delta V^{\prime \prime}\left(M^{2}\right) .
$$

This counterterm depends on the background only at $\mathrm{Q}\left(\lambda^{3}\right)$ order. If $\Delta V$ depends on the environment, these two counterterms define an environment dependent RS scheme.

If we denote by $\Delta V_{\mathrm{div}}^{\prime}$ and $\Delta V_{\mathrm{div}}^{\prime \prime}$ the divergent parts according to the $\overline{\mathrm{MS}}$ scheme, and by $\Delta V_{\overline{\mathrm{MS}}}^{\prime}=\Delta V^{\prime}-\Delta V_{\mathrm{div}}^{\prime}$ and $\Delta V_{\overline{\mathrm{MS}}}^{\prime \prime}=\Delta V^{\prime \prime}-\Delta V_{\mathrm{div}}^{\prime \prime}$ the finite parts [17], then we can write

$$
\begin{aligned}
& \delta \lambda_{\overline{\mathrm{MS}}}=-3 \lambda^{2} \Delta V_{\mathrm{div}}^{\prime \prime}\left(M^{2}\right) \\
& \delta m_{\mathrm{MS}}^{2}=-\lambda \Delta V_{\mathrm{div}}^{\prime}\left(M^{2}\right)+\frac{1}{2} \lambda^{2} \Phi^{2} \Delta V_{\mathrm{div}}^{\prime \prime}\left(M^{2}\right) .
\end{aligned}
$$

Using the renormalization scheme independence of the bare parameters we can relate the resummed and the $\overline{\mathrm{MS}}$ parameters at one loop level as

$$
\begin{aligned}
& \lambda-\lambda \overline{\mathrm{MS}}=3 \lambda^{2} \Delta V_{\overline{\mathrm{MS}}}^{\prime \prime}\left(M^{2}\right) \\
& m^{2}-m_{\overline{\mathrm{MS}}}^{2}=\lambda \Delta V_{\overline{\mathrm{MS}}}^{\prime}\left(M^{2}\right)-\frac{1}{2} \lambda^{2} \Phi^{2} \Delta V_{\overline{\mathrm{MS}}}^{\prime \prime}\left(M^{2}\right) .
\end{aligned}
$$


These equations determine the relation between the parameters of the RS scheme and of the $\overline{\mathrm{MS}}$ scheme; but it can also be interpreted as the formulae for how to resum diagrams in the $\overline{\mathrm{MS}}$ scheme. In fact, by the renormalization prescriptions of the RS scheme, $\lambda$ is the complete static 4-point function and $m^{2}$ is the complete mass.

The gap equations (33) are derived at one loop level, and are not necessarily useful at higher orders, that is it does not necessarily resum any subset of diagrams. There are arguments, however, that help to guess the correct form. To achieve super-daisy resummation, for example, we should use the resummed mass in the one loop expressions: that suggest that we should always use $m^{2}$ in the right hand side of (33) and $\lambda$ if it is multiplied with $\Phi^{2}$. Then all the possible mass resummations are done, so we should use $\lambda_{\overline{\mathrm{MS}}}$ for any other appearance of the coupling constant in the expression of the mass resummation. For the explicit coupling constants in the $\lambda$ resummation we recall that the double scoop (two bubbles) diagram contributes $3 \lambda \frac{3}{\mathrm{MS}}\left(\Delta V^{\prime \prime}\right)^{2}$, but if we iterate the expression for $\lambda$ as a function of $\lambda_{\overline{\mathrm{MS}}}$ in (33), we would get $6 \lambda_{\overline{\mathrm{MS}}}^{3}\left(\Delta V^{\prime \prime}\right)^{2}$. To cure this problem we should write $\lambda \lambda_{\overline{\mathrm{MS}}}$ instead of $\lambda^{2}$. So, finally, the proposed form for the resummation reads

$$
\begin{aligned}
& \lambda-\lambda_{\overline{\mathrm{MS}}}=3 \lambda \lambda_{\overline{\mathrm{MS}}} \Delta V_{\overline{\mathrm{MS}}}^{\prime \prime}\left(M^{2}\right) \\
& m^{2}-m_{\overline{\mathrm{MS}}}^{2}=\lambda_{\overline{\mathrm{MS}}} \Delta V_{\overline{\mathrm{MS}}}^{\prime}\left(M^{2}\right)-\frac{1}{2} \lambda \lambda_{\overline{\mathrm{MS}}} \Phi^{2} \Delta V_{\overline{\mathrm{MS}}}^{\prime \prime}\left(M^{2}\right),
\end{aligned}
$$

and $M^{2}=m^{2}+\frac{\lambda}{2} \Phi^{2}$, in agreement with 4 PI suggestions [14].

\section{A. Computation}

From (20) and (28) we find $\Delta V^{\prime}\left(M^{2}\right)=T_{B}\left(M^{2}\right) / 2$, and so we can write

$$
\begin{aligned}
& \Delta V_{\mathrm{MS}}^{\prime}\left(M^{2}\right)=\frac{M^{2}}{32 \pi^{2}} \ln \frac{M^{2}}{\mu^{2}}+\frac{1}{4 \pi^{2}} \int_{M}^{\infty} d \omega \sqrt{\omega^{2}-M^{2}} n(\omega) \\
& \Delta V_{\mathrm{MS}}^{\prime \prime}\left(M^{2}\right)=\frac{1}{32 \pi^{2}} \ln \frac{M^{2}}{\mu^{2}}-\frac{1}{8 \pi^{2}} \int_{M}^{\infty} \frac{d \omega}{\sqrt{\omega^{2}-M^{2}}} n(\omega) .
\end{aligned}
$$

It is instructive to examine these expressions for small masses. Since the result of the finite temperature part has $T / M$ as the only dimensionless quantity, this is equivalent to the high temperature approximation [1]

$$
\frac{1}{4 \pi^{2}} \int_{M}^{\infty} d \omega \sqrt{\omega^{2}-M^{2}} n(\omega)=\frac{T^{2}}{24}-\frac{T M}{8 \pi}-\frac{M^{2}}{32 \pi^{2}} \ln \frac{M^{2}}{c T^{2}}+\mathrm{Q}\left(\frac{M^{4}}{T^{2}}\right),
$$

where $\ln c=1+2 \ln 4 \pi-2 \gamma_{E} \approx 4.90762$. Then, using the notation $\mu^{2}=c \tilde{\mu}^{2}$, we have

$$
\begin{aligned}
& \Delta V_{\overline{\mathrm{MS}}}^{\prime}\left(M^{2}\right)=\frac{T^{2}}{24}-\frac{T M}{8 \pi}+\frac{M^{2}}{32 \pi^{2}} \ln \frac{T^{2}}{\tilde{\mu}^{2}} \\
& \Delta V_{\overline{\mathrm{MS}}}^{\prime \prime}\left(M^{2}\right)=-\frac{T}{16 \pi M}+\frac{1}{32 \pi^{2}} \ln \frac{T^{2}}{e \tilde{\mu}^{2}} .
\end{aligned}
$$

Then the gap equations read

$$
\begin{aligned}
& \frac{1}{\lambda}-\frac{1}{\lambda \overline{\mathrm{MS}}}=\frac{T}{16 \pi M}-\frac{3}{32 \pi^{2}} \ln \frac{T^{2}}{e \tilde{\mu}^{2}} \\
& m^{2}-m_{\overline{\mathrm{MS}}}^{2}=\frac{\lambda \overline{\mathrm{MS}} T^{2}}{24}-\frac{\lambda \overline{\mathrm{MS}} T}{8 \pi M}\left(M^{2}-\frac{\lambda}{4} \Phi^{2}\right)+\frac{\lambda \overline{\mathrm{MS}} m^{2}}{32 \pi^{2}} \ln \frac{T^{2}}{\tilde{\mu}^{2}}+\frac{\lambda \lambda_{\overline{\mathrm{MS}}} \Phi^{2}}{64 \pi^{2}} .
\end{aligned}
$$

The second term of the RHS of (39) depends on $\Phi$ at $\mathrm{Q}\left(\lambda^{3}\right)$ order, thus it is consistent to omit its background dependence. The last term is the consequence of the choice of the $\overline{\mathrm{MS}}$ counterterms. So we have

$$
\begin{aligned}
& \frac{1}{\lambda}-\frac{1}{\lambda \overline{\mathrm{MS}}}=\frac{T}{16 \pi M}-\frac{3}{32 \pi^{2}} \ln \frac{T^{2}}{e \tilde{\mu}^{2}} \\
& m^{2}-m_{\overline{\mathrm{MS}}}^{2}=\frac{\lambda \overline{\mathrm{MS}} T^{2}}{24}-\frac{\lambda \overline{\mathrm{MS}} T m}{8 \pi}+\frac{\lambda \overline{\mathrm{MS}} m^{2}}{32 \pi^{2}} \ln \frac{T^{2}}{\tilde{\mu}^{2}}+\frac{\lambda \lambda_{\overline{\mathrm{MS}}} \Phi^{2}}{64 \pi^{2}} .
\end{aligned}
$$


The solution therefore reads

$$
\begin{aligned}
& \lambda=\frac{\lambda \overline{\mathrm{MS}}}{1-\frac{3 \lambda_{\overline{\mathrm{MS}}}}{32 \pi^{2}} \ln \frac{T^{2}}{e \tilde{\mu}^{2}}+\frac{\lambda_{\overline{\mathrm{MS}}}}{16 \pi M}} \\
& m=\sqrt{U^{2}+W^{2}}-W
\end{aligned}
$$

where

$$
U^{2}=\frac{1}{\kappa}\left[m \frac{2}{\mathrm{MS}}+\frac{\lambda \overline{\mathrm{MS}} T^{2}}{24}+\frac{\lambda \lambda_{\overline{\mathrm{MS}}} \Phi^{2}}{64 \pi^{2}}\right], \quad W=\frac{\lambda \overline{\mathrm{MS}} T}{16 \pi \kappa}, \quad \kappa=1-\frac{\lambda \overline{\mathrm{MS}}}{32 \pi^{2}} \ln \frac{T^{2}}{\tilde{\mu}^{2}} .
$$

The solution has some remarkable properties. First of all it is a completely finite result, we do not need to bother with renormalization any more. Secondly - although coming from a high temperature expansion - it nicely extrapolates between the 4D and 3D fixed points of the system. For high scales, namely, (41) can be approximated as (we set $\Phi=0)$

$$
\begin{aligned}
& \lambda=\frac{\lambda_{\overline{\mathrm{MS}}}}{1-\frac{3 \lambda_{\overline{\mathrm{MS}}}}{32 \pi^{2}} \ln \frac{T^{2}}{e \widetilde{\mu}^{2}}} \\
& m^{2}=U^{2}=\frac{m_{\overline{\mathrm{MS}}}^{2}+\frac{\lambda \overline{\mathrm{MS}} T^{2}}{24}}{1-\frac{\lambda_{\overline{\mathrm{MS}}} \ln \frac{T^{2}}{32 \pi^{2}}}{\widetilde{\mu}^{2}}} .
\end{aligned}
$$

The formula for $\lambda$ is the result of the non-perturbative renormalization method of [15], where it was computed by the explicit summation of bubble diagrams.

We can realize here the running coupling and mass in the $\overline{\mathrm{MS}}$ scheme, and write

$$
\lambda=\lambda_{\overline{\mathrm{MS}}}(\tilde{T}), \quad m^{2}=m_{\overline{\mathrm{MS}}}^{2}(T)+\frac{\lambda_{\overline{\mathrm{MS}}}(T) T^{2}}{24},
$$

where $T^{2}=e \tilde{T}^{2}$. This corresponds to a $4 \mathrm{D}$ behavior in the $\overline{\mathrm{MS}}$ scheme.

At high temperatures the $T / m$ term takes over in the coupling constant, and the system follows a different trajectory. While the mass runs in the same way as before,

$$
\lambda=\frac{16 \pi m}{T} \approx 16 \pi \sqrt{\frac{\lambda_{\overline{\mathrm{MS}}}(T)}{24}} .
$$

This resummation describes correctly the second order nature of the phase transition (as opposed to the daisy resummation). Denoting $-m_{\overline{\mathrm{MS}}}^{2}=\frac{\lambda_{\overline{\mathrm{MS}}}}{24} T_{c}^{2}$, we find $m=0$ for $T=T_{c}$. Near the critical point the solution behaves as

$$
m=\frac{2 \pi}{3}\left(T-T_{c}\right), \quad \lambda=\frac{16 \pi m}{T_{c}} .
$$

The critical exponent for the mass is $\nu=1$ in this case, the fixed point of the coupling constant is $\lambda_{*}=0$ (ie. it becomes Gaussian), and the temperature dependence for the approach of this critical point is $\lambda-\lambda_{*} \sim\left(T-T_{c}\right)^{\kappa}$, where $\kappa=1$. This is exactly the behavior of the $O(N)$ model at $N \rightarrow \infty$ [16]. Indeed, in this simple approximation we resummed leading order diagrams in the large $N$ expansion, thus this agreement is not unexpected [1, 9$]$.

\section{CONCLUSION}

In this paper we investigated the question of to what extent the UV and IR regularization of a system, ie. renormalization and resummation can be reconciled. We described two methods: one is a constructive method of how to resum higher order counterterm diagrams in order to cure the UV divergences appearing in mass resummation in $\overline{\mathrm{MS}}$ scheme. Similarly to the thermal mass counterterm idea we introduced a "compensating counterterm" that has to be added to and subtracted from the original Lagrangian, the two terms contributing at different loop order. The other method is a rephrasing of this idea in the language of the renormalization group. Here we defined a new scheme, the resummation (RS) scheme, where the infinite part of the counterterms cancels the UV divergences, the finite part cancels the IR divergences of the theory at a given order. The drawback of this scheme is that it depends explicitly on 
the environment, but, with help of the renormalization group, we can project the results of the resummation scheme to a reference scheme, eg. $\overline{\mathrm{MS}}$. From the point of view of the resummation, this projection formally appears as a set of gap equations. We demonstrated these ideas by performing coupling constant and mass resummation in the $\Phi^{4}$ model.

In practice usually the problem of resummation and renormalization comes up in two forms. The first arises when we work on a diagrammatic basis and we compute a gap equation. At first glance the divergences of the resummed diagrams and counterterms do not cancel each other, so we wonder whether there is a method to consistently renormalize the divergent gap equations. We give positive answer to this question, proposing to use the compensating counterterm, or renormalization group ideas described in the paper. As a rule of thumb we can use that prescription that, for mass resummation in mass independent scheme, we first renormalize the equation perturbatively, and we apply this finite expression to compute the resummed parameters.

The other type of problem is when we modify our finite gap equation in a well motivated way, hoping that it catches important higher order effects, and we wonder whether the new form is compatible with renormalization. The answer is again positive: any form of the gap equation is compatible with renormalization. Technically we should treat our gap equations as transformation equations from $\overline{\mathrm{MS}}$ scheme to a specific RS scheme. Expansion of the solution of gap equations in terms of the coupling constants yields the finite difference of the counterterms in the two schemes at a given order. With the so-defined RS scheme we can do perturbation theory, that gives the possibility to systematically improve the resummation.

In the paper we treated only resummations of the parameters of the Lagrangian. As a future prospect we should investigate momentum dependent resummations, too. To extend our ideas we should reinterpret the renormalizable theories, allowing momentum dependence of the parameters.

\section{Acknowledgment}

The authors would like to thank András Patkós and János Polonyi for useful discussions. We acknowledge support from Hungarian Research Fund (OTKA) under contract numbers F043465, T034980 and T037689.

[1] L. Dolan and R. Jackiw, Phys.Rev. D 9 (1974) 3320.

[2] M. D'Attanasio and M. Pietroni, Nucl.Phys. B472 (1996) 711; D. Boyanovsky, H.J. de Vega and M. Simionato, Phys.Rev. D63 (2001) 045007

[3] E. Braaten and R. Pisarski, Nucl.Phys. B 337 (1990) 569.; B 339 (1990) 662.

[4] J.-P. Blaizot, E. Iancu and U. Reinosa, Nucl.Phys. A 736 (2004) 149; Phys.Lett. B 568 (2003) 016003; H. van Hees and J. Knoll, Phys.Rev. D 66 (2002) 025028; H.C. de Godoy Caldas, Phys.Rev. D 65 (2002) 065005.

[5] N. Banerjee and S. Mallik, Phys. Rev. D 43 (1991) 3368.

[6] A. Jakovác, A. Patkós, Zs. Szép and P. Szépfalusy, Phys. Lett. B 582 (2004) 179.

[7] J. Frenkel, A. Saa and J. Taylor, Phys.Rev. D 46 (1992) 3670; R. Parwani, Phys.Rev. D 45 (1994) 4965.

[8] S. Chiku and T. Hatsuda, Phys. Rev. D58, (1998) 076001; J.O. Andersen and M. Strickland, Phys.Rev. D 64 (2001) 105012.

[9] W. Buchmüller, Z. Fodor, T. Helbig and D. Walliser, Annals Phys. 234 (1994) 260.

[10] F. Karsch, A. Patkós, P. Petreczky, Phys.Lett. B401, 69-73, 1997

[11] J. Collins, Renormalization (Cambridge University Press, 1984.)

[12] D. O'Connor and C.R. Stephens, Int.J.Mod.Phys. A9 (1994) 2805; Erratum-ibid. A9 (1994) 5851.

[13] W. Buchmüller and O. Philipsen, Nucl.Phys. B443 (1995) 47.

[14] M.E Carrington, hep-ph/0401123 J. Bergess, hep-ph/0401172

[15] A. Patkós, Zs. Szép, P. Szépfalusy, Phys.Lett. B 537 (2002) 77

[16] S.-K. Ma, Phase transitions and critical phenomena (Academic Press, London, 1976, edited by C. Domb and M.S. Green)

[17] In $\overline{\mathrm{MS}}$ scheme $\left(\Delta V_{\mathrm{div}}^{\prime}\right)^{\prime} \neq \Delta V_{\mathrm{div}}^{\prime \prime}$ and so $\left(\Delta V_{\overline{\mathrm{MS}}}^{\prime}\right)^{\prime} \neq \Delta V_{\overline{\mathrm{MS}}}^{\prime \prime}$ 